\title{
Effect of fill-factor on the Talbot effect of diffraction gratings
}

\author{
Francisco Javier Salgado- \\ Remacha \\ fjsalgado@fis.ucm.es
}

Luis Miguel Sanchez-Brea

\section{Eusebio Bernabeu}

\begin{abstract}
Applied Optics Complutense Group, Optics Department Universidad Complutense de Madrid, Facultad de Ciencias Físicas, Ciudad Universitaria s.n., 28040, Madrid (Spain)
\end{abstract}

Applied Optics Complutense Group, Optics Department Universidad Complutense de Madrid, Facultad de Ciencias Físicas, Ciudad Universitaria s.n., 28040, Madrid (Spain)

Applied Optics Complutense Group, Optics Department Universidad Complutense de Madrid, Facultad de Ciencias Físicas, Ciudad Universitaria s.n., 28040, Madrid (Spain)

We analyze the effect of the fill factor of binary diffraction gratings on the Talbot effect under a scalar approach. We show that the location of the best-visibility planes changes with the configuration parameters of the grating, that is, the amplitude and phase modulation and the fill factor. Moreover, different intensity patterns and contrast shapes can be obtained when different configurations are used. Analytical expressions describing the contrast of the diffracted field are obtained, that agree with numerical simulations. [D0I: http: //dx.doi.org/10.2971/jeos.2011.11055]

Keywords: diffracting gratings, fill factor

\section{INTRODUCTION}

Diffraction gratings are key components in several fields as astrophysics, chemistry, biology, telecommunications, photonics engineering or medicine, and in a wide range of applications such as metrology, telescopes, spectroscopes, machine-tool, etc. [1]-[3]. Basically, a diffraction grating consists of a collection of reflecting or transmitting elements, separated periodically. The behavior of the grating in the far field is well studied under a wide range of conditions [4]-[6]. In this case, light propagates only at certain directions, known as diffraction orders. A model based on the Fourier approach allows us determining the directions of propagation, given by the well-known grating equation, and the power for each diffraction order [3], [4]. Since the angular separation of the diffraction orders strongly depends on the wavelength, low period diffraction gratings are especially relevant in spectroscopy, due to its ability to separate a polychromatic light into a spectrum in the far field [5].

However, in many applications diffraction gratings are used in Fresnel regime. Then, when the period of the grating is much longer than the wavelength, a Fresnel propagation based model is normally used to describe the behavior of gratings under a scalar approach [7]. Talbot effect rules the propagation of light: self-images appear at periodic distances from the grating, which are planes where the amplitude distribution is that of the field just after the grating. These planes are placed at fixed distances given by $z=l p^{2} / \lambda$, where $p$ is the period of the grating, $\lambda$ is the wavelength and $l$ is an integer.
When gratings are used to periodically modulate the amplitude of the field, such as in optical encoders, a maximum contrast is normally required [8]. In this sense, we can define the best visibility planes as the planes, parallel to the grating, in which the contrast is maximum, as it is defined below. The location of these best-visibility planes of the intensity patterns depends on the characteristics of the grating. For example, for amplitude gratings with a fill factor of $50 \%$ of the grating period this best-visibility planes are placed at Talbot planes $z=l p^{2} / \lambda$. On the other hand, for phase gratings with a fill factor of $50 \%$ of the grating period, the best visibility planes are placed at distances $z=\left(l+\frac{1}{2} p^{2} / \lambda\right)[7]$.

In a previous work, we have analyzed the self-imaging contrast of gratings with a mixed amplitude/phase character and its effects over the contrast under a scalar approach [9]. Nevertheless, the effect of the fill factor in the fringe formation in the scalar Fresnel regime has not been yet in-depth studied, to our knowledge. From the point of view of gratings manufacture, it is not always possible to fabricate gratings with a fill factor of exactly $50 \%$, especially when low periods are used. Sometimes, it results very difficult to delimit accurately the width of the grating strips.

In the present work, we will analyze the dependence of the diffracted intensity in the scalar near field with the fill factor for binary amplitude or phase diffraction gratings. In the first place, we have performed a numerical analysis based on the Beam Propagation Method, showing that there exists a strong 


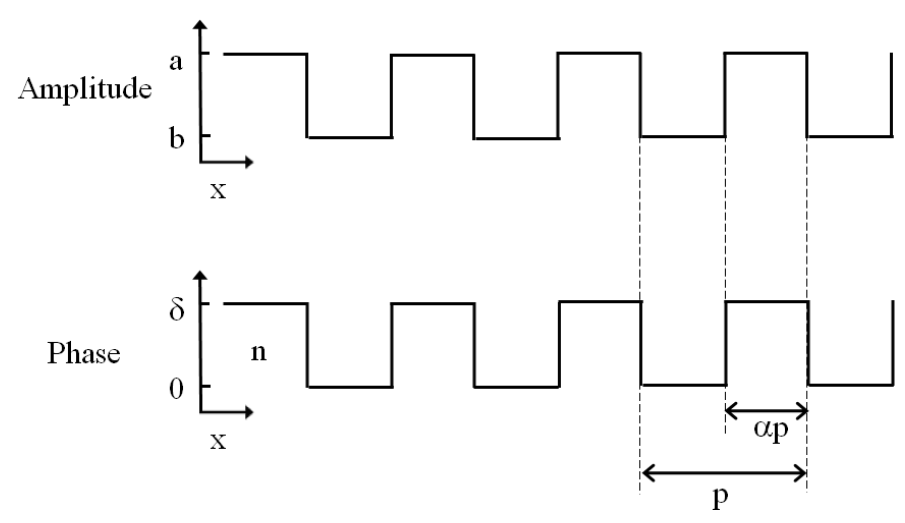

FIG. 1 Mixed amplitude-phase grating scheme with the involved parameters.

dependence between the fill factor of the grating and intensity pattern in the scalar Fresnel regime. After that, we will obtain simple equations for the intensity and contrast based on Fresnel approach using only the $-1^{\text {st }}, 0^{\text {th }}$ and $1^{\text {st }}$ orders. This approach is based on the fact that they contain more than $80 \%$ of the total intensity. Under this approach, we have found that the location of the best-visibility planes is not constant for phase gratings, but it varies in terms of the fill factor. Nevertheless, this approach does not explain all the effects we can find and, therefore, we have also performed an analytical approach with the orders between $-3^{\text {rd }}$ and $3^{\text {rd }}$. Then, the equations involved are not so simple, but we obtain a good description of the fill factor effects in the scalar Fresnel regime.

\section{Simulations by means of Beam Propagation Method}

Let us consider a binary grating whose fill factor can take values between $0 \%$ and $100 \%$ of the grating period. We describe with the same formalism both amplitude and phase gratings (and also mixed amplitude/phase gratings) [9]. The involved parameters are described in Figure 1. A plane wave with wavelength $\lambda$ illuminates a binary grating with period $p$ and fill factor. The parameters $a$ and $b$ represent the maximum and minimum value of the amplitude modulation, and the phase retardation produced by phase gratings is $\delta=2 \pi h(n-1) / \lambda$, where $n$ is the refractive index of the grating and $h$ is the height of the strips. Thus, for the case of ideal amplitude gratings $a=1, b=0$, and $\delta=0$; and for phase gratings $a=1, b=1$ and delay $\delta$. The grating is defined along $x$-axis, and the beam light propagates along $z$-axis. We consider that the period $p$ is much longer than the wavelength $\lambda$. Then, a scalar approach on the paraxial domain is valid.

In the first place, we have developed numerical simulations based on the Beam Propagation Method (BPM), a numerical method for the simulation of light propagation under a scalar approach taking into account a refractive index map [10]. Originally, it was proposed for the simulation of waveguides with variable refractive index [11], but it is also successfully used for the simulation of gratings [12], liquid crystal devices
[13] and, in general, photonics devices with complex analytical description [14]. In our case, the algorithm consists of a Fourier-Transform-based Fresnel propagator, considering light under paraxial and scalar domain. Usually, Berenguers Perfectly Matched Layers (PML) approach is used to avoid reflections at the border of the computation window [15], [16]. Instead, we prefer to use a super-Gaussian absorbing profile defined along $x$ and $y$ directions, obtaining a non-absorbing flat zone in the central propagation area, and high-absorbing zones at the borders of the propagation window. Then, the amplitude at the borders is avoided and, consequently, the spurious boundaries reflections are also avoided.

For the simulations, we use two steps. First, we propagate the illumination field (a plane wave with normalized amplitude) through a diffracting grating with the proper parameters: amplitude, phase, period, and fill factor. The resulting field, at the end of the grating, is taken as illumination field for the last propagation, along vacuum space. Then, the origin at the $\mathrm{z}$-axis is located at the end of the grating. In this way, we obtain different resolutions for the propagation through the grating (with normally a small depth) and for the propagation through free space. We calculate the intensity in each point of the space using $\hat{I}(x, z)=U(x, z) U^{*}(x, z)$, where $U(x, z)$ and $U^{*}(x, z)$ denote the field and the conjugated field for each point of the space provided by BPM. To analyze the visibility of the fringes produced by the grating in the near field we use the definition of contrast along z-axis

$$
\operatorname{Contrast}(z)=\left|\frac{I_{\max }-I_{\min }}{I_{\max }+I_{\min }}\right|,
$$

where $I_{\max }$ is the intensity at $x=0$ for every value of $z$, $I_{\max }=I(x=0, z)$, and $I_{\min }$ is the intensity at $x=p / 2$ for every value of $z, I_{\min }=I(x=p / 2, z)$. Due this definition, where the absolute value has been used, odd and even selfimages are equivalent and we do not consider the inversion of contrast (in other words, the contrast is always positive).

In Figure 2 we have plotted the diffracted intensity in the near field, and also the contrast obtained with Eq. 1 for BPM simulations of an amplitude grating $(a=1, b=0, \delta=0)$ with $p=40 \mu \mathrm{m}$ for the cases of $\alpha=30 \%, \alpha=50 \%$, and $\alpha=70$ $\%$, illuminated with $\lambda=632.8 \mathrm{~nm}$. For all the simulations performed in this work we have used 2048 pixels along the direction of propagation, and we have propagated until $4 z_{T}$, where $z_{T}=p^{2} / \lambda=2.53 \mu \mathrm{m}$ is known as the Talbot distance. Thus, the distance of propagation is $10.12 \mu \mathrm{m}$. At the same time, the transversal width is $1 \mathrm{~mm}$ of with the same number of pixels. As it can be observed, the location of the best-visibility planes is the same for the three cases. Also, we can see that when a grating with narrow transparent strips along $x$-axis (corresponding to a low fill factor) is used, the contrast along $z$-axis has the form wide maxima peaks at the location of the Talbot planes.

On the contrary, when a grating with wide transparent strips (corresponding to a high fill factor) is used, the contrast along 


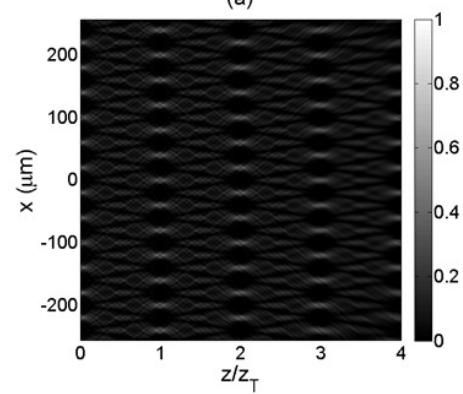

(c)

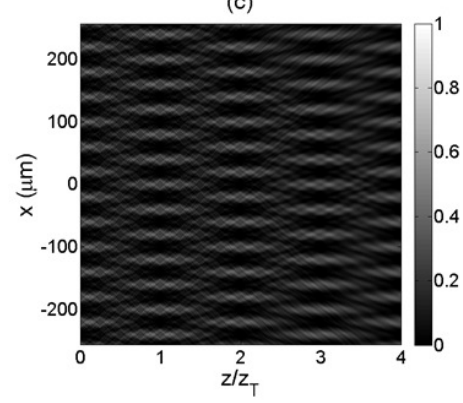

(e)

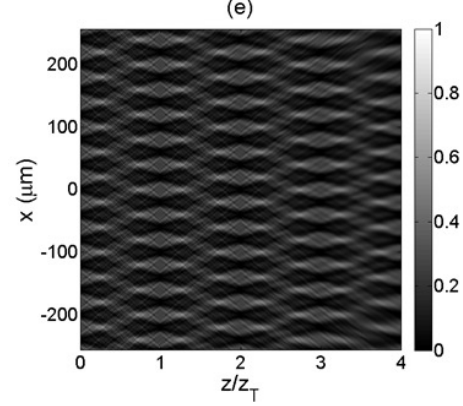

(b)

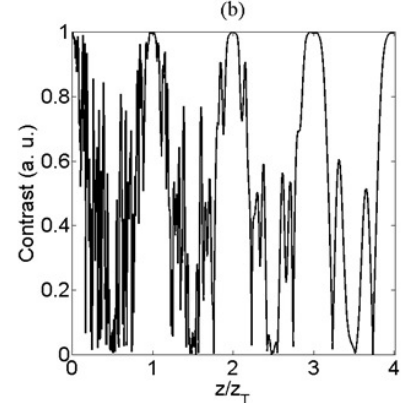

(d)
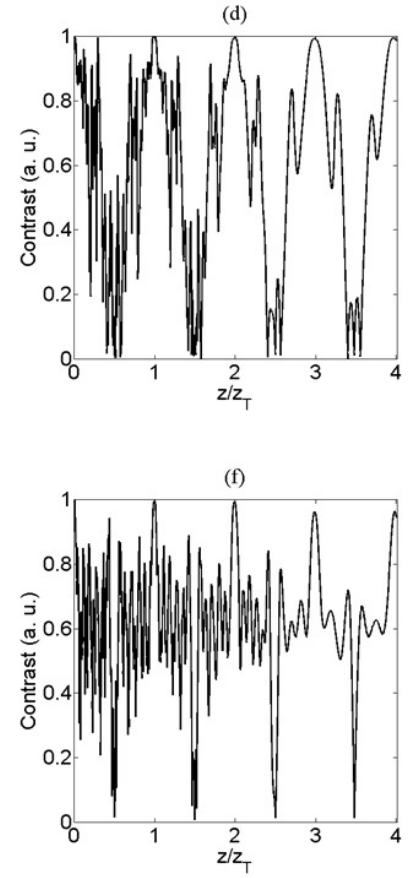

FIC. 2 Intensity (logarithmic scale) and contrast obtained by means of BPM simulations for a grating with $p=40 \mu \mathrm{m}, a=1, b=0$ and $\delta=0$, illuminated with $\lambda=632.8$ $\mathrm{nm}$, for different values of $\alpha$; a) and b) $\alpha=30 \%$ of the grating period; c) and d) $\alpha=50 \%$ of the grating period; e) and f) $\alpha=70 \%$ of the grating period.

$z$-axis is formed by narrow maxima peaks, separated each other by a Talbot distance, over an almost-stable value (but a rippled function). A stable high value of the contrast over a wide region along $z$-axis is interesting for applications in which self-images are used, increasing the mechanical positioning tolerances.

In order to reach a more generalized point of view, let us compare an amplitude $(a=1, b=0, \delta=0)$ and a phase grating $(a=1, b=1, \delta=\pi / 2)$, both with period $p=40 \mu \mathrm{m}$ and illuminated with $\lambda=632.8 \mathrm{~nm}$, over a wide range of values of the fill factor $\alpha$. The results are shown in Figure 3. The values of the contrast are now collected at the same figure for each kind of grating, where the vertical axis represent the value of $\alpha$. These plots are normalized to its maximum value. It should be mentioned that these figures show the contrast of the intensity patterns, and not the absolute intensities. In terms of global intensity amount, for a phase grating there is no dependence with $\alpha$, whereas for an amplitude grating the amount of intensity that pass
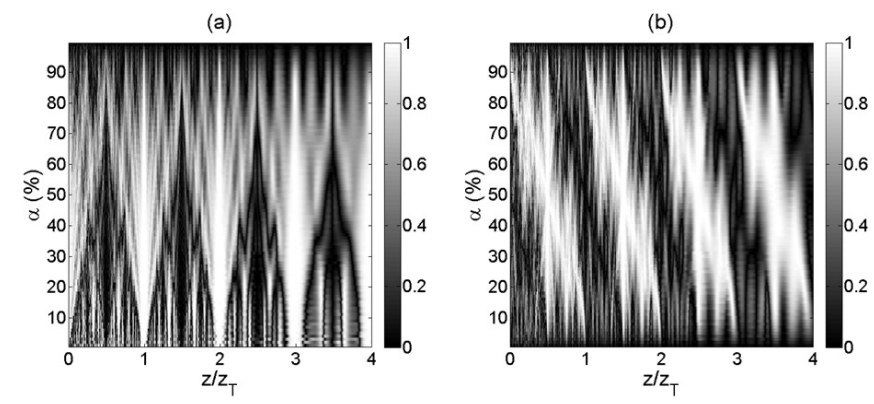

FIG. 3 Dependence of contrast obtained using BPM with $\alpha$ (in \% of the grating period) for a grating with $p=40 \mu \mathrm{m}$, and a) $\mathrm{a}=1, \mathrm{~b}=0$ and $\delta=0$; and b) $\mathrm{a}=1, \mathrm{~b}=1$, $\delta=\pi / 2$, illuminated with $\lambda=632.8 \mathrm{~nm}$.

through the grating depends strongly with the fill factor.

It should be here noted that, since we have used a scalar propagator, the range of validity of our calculations are limited to the region in which the typical feature size is longer than $4 \lambda$. Since the wavelength used is $\lambda=632.8 \mathrm{~nm}$, the minimum strip width that can be considered is about $2 \mu \mathrm{m}$, corresponding to a fill factor of $2 \mu \mathrm{m}$ about $6 \%$. Thus, the range of validity of these simulations is between $6 \%$ and $94 \%$.

The dependence with $\alpha$ is notorious and different for amplitude and phase gratings. For an amplitude grating the width of the best-visibility planes along $z$-axis tends to increase with $\alpha$. The location of the maximum values, as we have seen, remains the same for every value of $\alpha$. On the contrary, the location of this maxima for a phase grating moves along a Talbot period when $\alpha$ changes, whereas the shape of the bestvisibility planes remains almost constant along z-axis. Up to our knowledge, this behavior (specially, the change of the location of the best-visibility planes when the fill factor changes) has not been yet explained. Therefore, we find necessary an analytical study of this phenomenology.

\section{Theoretical Model}

Since diffraction gratings are periodical elements, they can be described as a series expansion. According to Thin Element Approximation (TEA) [17], the transmittance of the grating is given by

$$
t(\xi)=\sum_{l} c_{l} \exp (i q l \xi)
$$

where $q=2 \pi / p$ and $c_{l}$ are the Fourier coefficients of the grating, defined as [9]

$$
\begin{aligned}
& c_{0}=\alpha\left(a e^{-i \delta}-b\right)+b, \\
& c_{l}=\alpha\left(a e^{-i \delta}-b\right) \operatorname{sinc}(\pi l \alpha),
\end{aligned}
$$

The efficiency of each order of the grating is determined by these coefficients, according to abs $\left(c_{l} c_{l}^{*}\right)$ (where $c_{l}^{*}$ means complex conjugated). A scalar treatment of the diffraction is possible when the period of the grating is much longer than the wavelength of the incident beam. In our case, attending to 
optical encoders, the period of the common gratings are in the range of $20-40 \mu \mathrm{m}$ and the wavelength in the visible region, so the scalar approach is possible. It should be noticed that this scalar treatment will be valid only if the wide of the grating strips are much longer than the wavelength, as in the case of the BPM simulations. In other words, our development loss validity when the fill factor is lower than $6 \%$ or higher than $94 \%$ (taking into account periods of $40 \mu \mathrm{m}$ and $\lambda=632.8 \mathrm{~nm}$ ). The diffracted field at a distance $z$ from the grating is then described using the Fresnel approach,

$$
U(x, z)=\frac{\exp (i k z)}{\sqrt{i \lambda z}} \int U_{0}(\xi) t(\xi) \exp \left[i \frac{k}{2 z}(x-\xi)^{2}\right] d \xi,
$$

where $U_{0}(\xi)$ is the illumination wave. When a monochromatic plane wave $U_{0}(\xi)=A_{0}$ illuminates the diffraction grating, the diffracted field at a distance $z$ from the grating results

$$
U(x, z)=A_{0} \sum_{l} c_{l} \exp (i q x l) \exp \left(i \pi l^{2} z / z_{T}\right) .
$$

At distances multiples of $z_{T}$, self-images of the grating are produced. The normalized intensity of the diffracted field, $\hat{I}(x, z)=U(x, z) U^{*}(x, z) /\left|A_{0}\right|^{2}$, results in

$$
\hat{I}(z, x)=\sum_{l} \sum_{l^{\prime}} c_{l} c_{l^{\prime}}^{*} \exp \left[i q x\left(l-l^{\prime}\right)\right] \exp \left[i \pi\left(l^{2}-l^{\prime 2}\right) \hat{z}\right],
$$

where $\hat{z}=z / z_{T}$ and ${ }^{*}$ denotes complex conjugated. The main consequence of this equation is that maxima of intensity appear at certain distances after the grating, separated by $z_{T}$, as we have seen in the simulations. In general, these Fourier coefficients are complex variables, and they are written as $c_{l}=C_{l} \exp \left(i \beta_{l}\right)$, being $C_{l}$ their modulus and $\beta_{l}$ their phase. Then Eq. 6 can be rewritten as

$$
\begin{aligned}
\hat{I}(z, x)= & \sum_{l} \sum_{l^{\prime}} C_{l} C_{l^{\prime}} \exp \left[i q x\left(l-l^{\prime}\right)\right] \\
& \times \exp \left[i \pi\left(l^{2}-l^{\prime 2}\right) \hat{z}+i\left(\beta_{l}-\beta_{l^{\prime}}\right)\right] .
\end{aligned}
$$

The accuracy of this analytical development depends on the number of orders taken into account. On the other hand, a high number of orders implies a high complexity in the final expressions. For a rough estimation of the intensity distribution at the self-images, we can use at a first stage only the $1^{\text {st }}, 0^{\text {th }}$ and $1^{\text {st }}$ orders. In Figure 4 , the cumulative efficiency of the grating (using the Fourier coefficients in Eq. 7), taking into account different number of orders, is plotted against the fill factor for an amplitude grating and for a phase grating. In the case of an amplitude grating with $a=1$ and $b=0$ the total efficiency should be linear with $\alpha$ (since the total energy that can pass through the grating depends linearly with $\alpha$ ). On the other hand, for a phase grating the total efficiency should be always equal to unity. As it can be observed, the $-1^{\text {st }}, 0^{\text {th }}$ and $1^{\text {st }}$ orders carry more than $80 \%$ of the energy for both kinds of gratings. This approach has been successfully used to determine the behavior of diffraction gratings under a scalar Fresnel approach for a wide range of circumstances [19], [20].

When only $-1^{\text {st }}, 0^{\text {th }}$ and $1^{\text {st }}$ orders are considered, a simple equation for the intensity is obtained, and Eq. 7 simplifies to

$$
\begin{aligned}
\hat{I}(x, z) \approx & C_{0}^{2}+4 C_{1}^{2} \cos (q x)^{2} \\
& +4 C_{0} C_{1} \cos (q x) \cos \left(\pi \hat{z}+\beta_{0}-\beta_{1}\right) .
\end{aligned}
$$

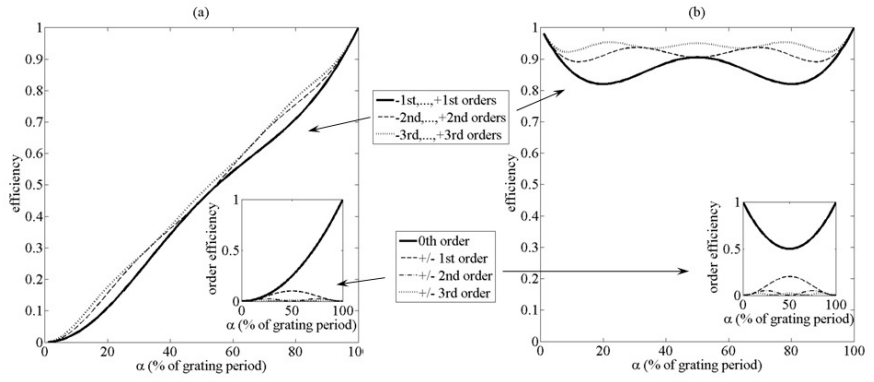

FIG. 4 Cumulative efficiency of the grating against fill factor, taking into account up to the $\pm 1^{\text {st }}$ (continuous line), $\pm 2^{\text {nd }}$ (dashed line) and $\pm 3^{\text {rd }}$ (plotted line) diffracting orders (subplot: efficiency of each order for different values of $\alpha$ ), a) pure amplitude grating with $\mathrm{a}=1, \mathrm{~b}=0$ and $\delta=0$; and $\mathrm{b}$ ) phase grating with $\mathrm{a}=1, \mathrm{~b}=1, \delta=\pi / 2$.
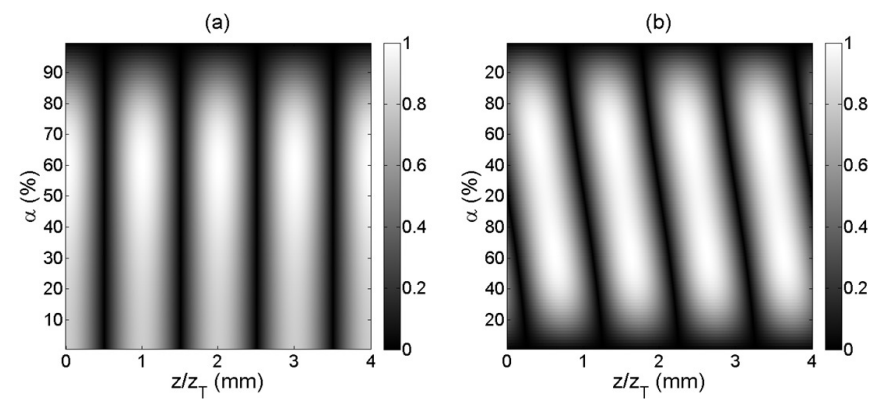

FIG. 5 Dependence of contrast (absolute value) with using up to the $-1^{\text {st }}, 0^{\text {th }}$ and $1^{\text {st }}$ orders for a) a pure amplitude grating with $\mathrm{a}=1, \mathrm{~b}=0$ and $\delta=0$; and $\mathrm{b}$ ) a phase grating with $\mathrm{a}=1, \mathrm{~b}=1, \delta=\pi / 2$.

Thus, the contrast results in

$$
\text { Contrast }_{ \pm 1}(z)=\frac{4 C_{0} C_{1}}{C_{0}^{2}+4 C_{1}^{2}}\left|\cos \left(\pi \hat{z}+\beta_{0}-\beta_{1}\right)\right| .
$$

For the case of an amplitude grating, the Fourier coefficients are $c_{0}=\alpha, c_{1}=c_{-1}=\alpha \operatorname{sinc}(\pi \alpha)$ then the coefficients $\beta_{0}=\beta_{1}=0$ are null. However, this is not the case for phase gratings. For example, the Fourier coefficients for a $\pi / 2$ phase grating are $c_{0}=\sqrt{2 \alpha^{2}-2 \alpha+1} \exp [-i \alpha /(1-\alpha)]$, and $c_{1}=\alpha \sqrt{2} \operatorname{sinc}(\pi \alpha) \exp (3 \pi i / 4)$. Consequently, there appears a shift in the location of the self-images which depends on the fill factor according to

$$
\beta_{0}-\beta 1=\frac{-\alpha}{1-\alpha} \frac{3 \pi}{4}
$$

The intensity distribution in the near field for these two particular cases (the amplitude grating and the $\pi / 2$ phase grating) is shown in Figure 5. Attending to these figures, we notice that Eq. 9 explains the location of the maxima of contrast: a change in the location of the self-images is appreciable for the phase grating, but not for the amplitude grating. Nevertheless, a visual comparison between Figure 3 and Figure 5 shows that Eq. 9 does not predict completely the behavior of the grating in the Fresnel regime, especially in terms of contrast pattern shape. These effects can be attributed to the energy carried by upper orders, shown in Figure 4 . When the fill factor is higher or lower than $50 \%$ of the grating period, the upper orders $\left( \pm 2^{\text {nd }}, \pm 3^{\text {rd }}\right)$ reach a higher percentage in the energetic distribution of Figure 4.

In order to evaluate the number of orders needed, we solve numerically Eq. 7 for amplitude gratings, since the effect is 


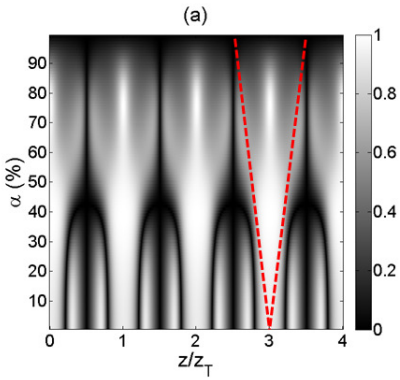

(c)

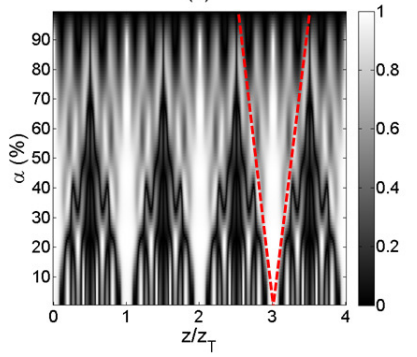

(b)

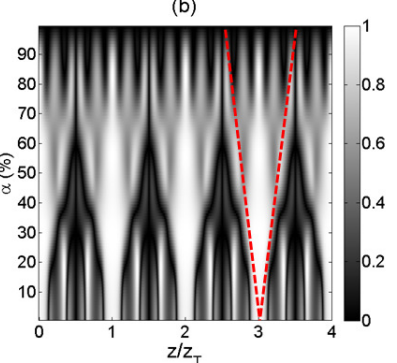

(d)

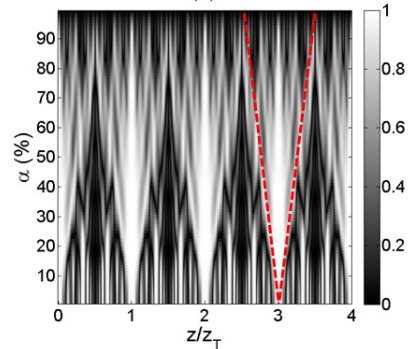

FIG. 6 Dependence of the contrast with for pure amplitude gratings with $a=1$, $\mathrm{b}=0, \delta=0$ and $\mathrm{p}=40 \mu \mathrm{m}$ illuminated with $\lambda=632.8 \mathrm{~nm}$, taking into account different number of orders; a) using up to the $\pm 2^{\text {nd }}$ orders; b) using up to the $\pm 3^{\text {rd }}$ orders; c) using up to the $\pm 4^{\text {th }}$ orders; and d) using up to the $\pm 5^{\text {th }}$ orders.

visually more obvious for this case, taking into account different number of orders. The results are shown in Figure 6. It is clear that the inclusion of the $\pm 2^{\text {nd }}$ orders is enough to predict the dependence between the fill factor and best-visibility planes width. For a higher number of orders, rippled signals with spatial periodicity lower than $z_{T}$ appear over the fundamental contrast signal with periodicity $z_{T}$. These higher frequencies are more visible for high fill factors (over 80\%) and low fill factors (under 20\%). Therefore, very narrow peaks on the contrast profiles can be obtained using high or low fill factors. Moreover, the width of the best-visibility planes tends, when the number of orders involved increases, to a linear dependence with $\alpha$. This dependence is shown with dashed lines over plots of Figure 6. Assuming that self-images width, $W_{s}$, is the distance between points at $\mathrm{z}$-axis where the contrast fall down to half-height, we can write,

$$
W_{s} \approx \alpha,
$$

which can be a useful expression for diffraction grating design. For some applications using self-imaging effects, it is important to obtain wide areas with high contrast along $z-$ axis, as in optical encoders. Attending to the plots in Figure 6 , we notice that using up to $\pm 3^{\text {rd }}$ orders we can obtain a description of both effects: the secondary peaks from extreme fill factors, and the almost-linearly dependence between $W_{s}$ and $\alpha$. The inclusion of a higher number of orders can complicate the analytical development.

Developing Eq. 7 up to $\pm 3^{\text {rd }}$ orders, and taking into account that $C_{l}=C_{-l}$ (since sinc is an even function), then the inten-
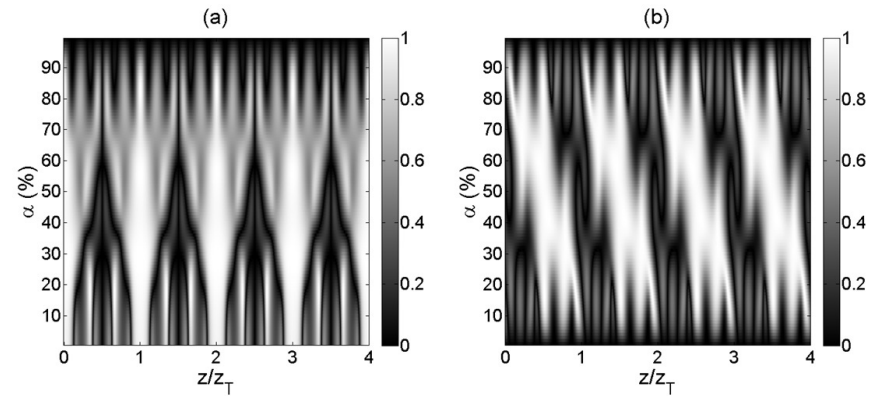

FIC. 7 Analytical contrast obtained taking into account up to the $\pm 3^{\text {rd }}$ orders for different values of $\alpha$, for a grating with $\mathrm{p}=40 \mu \mathrm{m}$, and a) a pure amplitude grating with $\mathrm{a}=1, \mathrm{~b}=0$ and $\delta=0$; and $\mathrm{b}$ ) a phase grating with $\mathrm{a}=1, \mathrm{~b}=1, \delta=\pi / 2$; illuminated with $632.8 \mathrm{~nm}$.

sity results

$$
\begin{aligned}
\hat{I}(z, x) \approx & C_{0}^{2}+4 C_{1}^{2} \cos (q x)^{2}+4 C_{2}^{2} \cos (2 q x)^{2} \\
& +4 C_{3}^{2} \cos (3 q x)^{2} \\
& +4 C_{0} C_{1} \cos (q x) \cos \left(\pi \hat{z}-\beta_{0}+\beta_{1}\right) \\
& +4 C_{0} C_{2} \cos (2 q x) \cos \left(4 \pi \hat{z}-\beta_{0}+\beta_{2}\right) \\
& +4 C_{0} C_{3} \cos (3 q x) \cos \left(9 \pi \hat{z}-\beta_{0}+\beta_{3}\right) \\
& +8 C_{1} C_{2} \cos (q x) \cos (2 q x) \cos \left(3 \pi \hat{z}-\beta_{1}+\beta_{2}\right) \\
& +8 C_{1} C_{3} \cos (2 q x)^{2}[2 \cos (2 q x)-1] \\
& \times \cos \left(8 \pi \hat{z}-\beta_{1}+\beta_{3}\right) \\
& +4 C_{2} C_{3}[\cos (q x)+\cos (5 q x)] \\
& \times \cos \left(5 \pi \hat{z}-\beta_{2}+\beta_{3}\right),
\end{aligned}
$$

and the contrast results in

$$
\begin{array}{cc} 
& 4 C_{0} C_{1} \cos \left(\pi \hat{z}-\beta_{0}+\beta_{1}\right) \\
& +8 C_{1} C_{2} \cos \left(3 \pi \hat{z}-\beta_{1}+\beta_{2}\right) \\
& +8 C_{2} C_{3} \cos \left(5 \pi \hat{z}-\beta_{2}+\beta_{3}\right) \\
\text { Contrast }_{ \pm 3}(z)=\quad & +4 C_{0} C_{3} \cos \left(9 \pi \hat{z}-\beta_{0}+\beta_{1}\right) \\
\hline C_{0}^{2}+4 C_{1}^{2}+4 C_{2}^{2}+4 C_{3}^{2} \\
+4 C_{0} C_{2} \cos \left(4 \pi \hat{z}-\beta_{0}+\beta_{2}\right) \\
+4 C_{1} C_{3} \cos \left(8 \pi \hat{z}-\beta_{1}+\beta_{3}\right) .
\end{array}
$$

The numerical solutions of Eq. 13 for a pure amplitude grating and for a $\pi / 2$ phase grating (with the proper Fourier coefficients) has been plotted in Figure $7 \mathrm{a}$ and Figure $7 \mathrm{~b}$ respectively, for different values of $z$ and $\alpha$. The concordance with the BPM simulations is clear. We also note that the central region (between $20 \%$ and $70 \%$ ) in both cases lead to a stable region, with no secondary peaks. For the case of optical encoding, the most attractive case should be an amplitude grating rounding $\alpha=70 \%$, since the width of the maximum peaks are enlarged.

We can take some heuristic considerations from Eq. 13. Some cosine functions appear with different frequencies, $z_{T}, z_{T} / 3$, $z_{T} / 5$, and $z_{T} / 9$. The arguments of the Fourier coefficients of the grating, $\beta_{l}$, introduce a displacement in the maxima of each cosine function. For the case of an amplitude grating, the Fourier coefficients are $c_{0}=\alpha, c_{1}=\alpha \operatorname{sinc}(\pi \alpha)$, $c_{2}=\alpha \operatorname{sinc}(2 \pi \alpha)$ and $c_{3}=\alpha \operatorname{sinc}(3 \pi \alpha)$, and consequently the arguments $\beta_{l}$ are null. Therefore there is not displacement of the maxima with $\alpha$. 
Disregarding the displacement introduced by each $\beta_{l}$, the numerator in Eq. 13 is a sum of cosine functions with frequencies $z_{T}, z_{T} / 3, z_{T} / 5$, and $z_{T} / 9$. The absence of even spatial frequencies in the numerator ensures a minimum of contrast at multiplies of $z_{T} / 2$ and a maximum at multiplies of $z_{T}$. The final contrast can be understood as a sum of cosines with different weights. Depending on the factors multiplying each cosine function, the smaller frequencies can be more visible than the frequency $z_{T}$, as occurs for high and low $\alpha$. Under some circumstances, the sum of cosine functions produces an enlarging of $W_{s}$ with $\alpha$.

For a phase grating the $0^{\text {th }}$ Fourier coefficients is

$$
c_{0}=\sqrt{2 \alpha^{2}-2 \alpha+1} \exp [-\alpha i /(1-\alpha)]
$$

and therefore $\beta_{0}$ introduces a displacement in the maxima with a linear dependence with $\alpha$.The upper coefficients are

$$
\begin{aligned}
& c_{1}=\alpha \sqrt{2} \operatorname{sinc}(\pi \alpha) \exp [3 \pi i / 4], \\
& c_{2}=\alpha \sqrt{2} \operatorname{sinc}(2 \pi \alpha) \exp [3 \pi i / 4], \text { and } \\
& c_{3}=\alpha \sqrt{2} \operatorname{sinc}(3 \pi \alpha) \exp [3 \pi i / 4] .
\end{aligned}
$$

Thus, these upper coefficients are not necessary to explain the linear dependence of the maxima location with $\alpha$, but they introduce some higher spatial frequencies in the self-imaging process. It should be also mentioned that we have not considered the different efficiency of an amplitude grating and a phase grating, shown in Figure 4.

We can extract some conclusions. For lower values of $\alpha$, the best-visibility planes are almost peaks centered on the position of the Talbot planes. Secondary maxima appear for the low and high values of $\alpha$. However, for the study of these extreme regions a vectorial approach should be necessary. The dependence of the shape can be understood as a sum of cosine functions with odd spatial frequencies. Nevertheless, the location of the maxima of contrast does not change. On the contrary, for the $\pi / 2$ phase grating case shown in Figure 2(b) we can appreciate the strong dependence of the location of the maximum contrast with the fill factor, whereas the self-images width do not change considerably.

The application of this development results clear when we think of the tolerances analysis of systems with diffraction gratings. In this work we have seen that deviation from fill factor of $50 \%$ can involve changes in the maxima location and in the contrast shape, which should be taken into account. From a more generalized point of view, the proper election of the parameters involved in Eq. 13 can be used for the optimization of the contrast shape. For example, it could be possible to maximize the maxima of contrast width, which result really interesting for optical encoders.

\section{Conclusions}

We have studied the effect of the fill factor over the near field of diffraction gratings under a scalar approach. We have found, by means of BPM simulations, that the location of the best-visibility planes do not change for pure amplitude grating when the fill factor changes, whereas for phase gratings the location of best visibility planes changes linearly with the fill factor. Moreover, for amplitude gratings the width of the maxima of contrast tends to a linear dependence with $\alpha$.

An analytical development under a scalar approach considering the $-1^{\text {st }}, 0^{\text {th }}$ and $1^{\text {st }}$ orders can predict the displacement of the best-visibility planes. Nevertheless, for a proper description of the effects, it is necessary to develop up to the $3^{\text {rd }}$ orders.

Then, we can describe the dependence of the width and shape of the best-visibility planes with $\alpha$ for amplitude gratings. For low and high fill factor, new secondary maxima appear between consecutive best-visibility planes. For the case of phase gratings, there is a displacement of the best-visibility, and secondary maxima appear again for low and high fill factor values. This final result can be understood as the competence between several cosine functions with different spatial frequencies, whose weights change with $\alpha$. The expression found can be used to improve the tolerances of systems based on Talbot effect.

\section{Acknowledgements}

This work has been partially supported by project DPI201127851 and project INNPACTO "FORE", IPT-020000-2010-9 of the Ministerio de Ciencia e Innovación of Spain.

\section{References}

[1] E. G. Loewen, and E. Popov, Diffraction gratings and applications (Marcel Dekker, New York, 1997).

[2] C. Palmer, Diffraction Grating Handbook (Richardson Grating Laboratory, New York, 2000).

[3] M. Born, and E. Wolf, Principles of Optics (Pergamon Press, Oxford, 1980).

[4] J. W. Goodman, Introduction to Fourier Optics (McGraw-Hill, New York, 1968).

[5] C. 0. Reynolds, J. B. DeVelis, G. B. Parrent Jr., and B. J. Thompson, The New Physical Optics notebooks tutorials in Fourier Optics (SPIE Press, Bellingham, 1989).

[6] D. C. O’Shea, T. J. Suleski, A. D. Kathman, and D. W. Prather Diffractive Optics. Design, Fabrication, and Test (SPIE Press, Bellingham, 2003).

[7] K. Patorski, "The self imaging phenomenon and its applications", Prog. Optics 27, 3-108 (1989).

[8] L.M. Sanchez-Brea, and T. Morlanes, "Metrological errors in optical encoders", Meas. Sci. Technol. 19(11), 115104 (2008).

[9] F. J. Torcal-Milla, L. M. Sanchez-Brea, and F. J. Salgado-Remacha, "Self-images location of amplitude/phase binary gratings", App. Optics 48, 6252-6258 (2009).

[10] M. D. Feit, and J. A. Fleck Jr., "Light propagation in graded-index optical fibers" App. Optics 17, 3990-3998 (1978). 
[11] M. Koshiba, Y. Tsuji, and M. Hikari, "Finite Element Beam Propagation Method with Perfectly Matched Layer Boundary Conditions", IEEE T. Magn. 35, 1482-1485 (1999).

[12] D. Yevick, and L. Thylén, "Analysis of gratings by the beampropagation method" J. Opt. Soc. Am. 72, 1084-1089 (1982).

[13] P. J. M. Vanbrabant, J. Beeckman, K. Nayts, R. James, and F. A. Fernandez, "A finite element beam propagation method for simulation of liquid crystal devices" 0pt. Express 17, 10895-10909 (2009).

[14] P. Srisungsitthisunti, 0. K. Ersoy, and X. Xu, “Beam propagation method modeling of modified volume Fresnel zone plates fabricated by femtosecond laser direct writing" J. Opt. Soc. Am. A 26, 188-194 (2009).

[15] J. P. Berenguer, "A perfectly matched layer for the absorption of electromagnetic waves" J. Comput. Phys. 114, 185-200 (1994).

[16] W. P. Huang, C. L. Xu, W. Lui, and K. Yokoyama, "The perfectly matched layer (PML) boundary condition for the beam propagation method" IEEE Photonic. Tech. L. 8, 649-651 (1996).

[17] U. Levy, E. Marom, and D. Mendlovic, "Thin element approximation for the analysis of blazed gratings: simplified model and validity limits" Opt. Commun. 229, 11-21 (2004).

[18] E. Keren, and 0. Kafri, "Diffraction effects in moiré deflectometry" J. Opt. Soc. Am. A 2, 111-120 (1985).

[19] F. J. Torcal-Milla, L. M. Sanchez-Brea, F. J. Salgado-Remacha, and E. Bernabeu, "Self-imaging with curved gratings" Opt. Commun. 283(2) 3869-3873 (2010).

[20] L. M. Sanchez-Brea, J. Saez-Landete, J. Alonso, and E. Bernabeu, "Invariant grating pseudoimaging using polychromatic light and a finite extension source" Appl. Optics 47(10), 1470-1477 (2008). 